\title{
Preferência manual numa tarefa de antecipação-coincidência: efeitos da direcção do estímulo
}

\author{
Paula C. Rodrigues ${ }^{1}$ \\ Cidália Freitas ${ }^{1}$ \\ Maria Olga Vasconcelos ${ }^{1}$ \\ João Barreiros²
}

https://doi.org/10.5628/rpcd.07.01.109

\author{
${ }_{1}^{1}$ Faculdade de D esporto \\ Universidade do Porto \\ Portugal \\ 2 Faculdade de M otricidade H umana \\ Universidade Técnica de Lisboa \\ Portugal
}

\section{RESUMO}

Neste estudo objectivamos analisar a relação existente entre a performance numa tarefa de antecipação-coincidência, variando a orientação do estímulo, a preferência manual e o sexo. Participaram 31 destrímanos e 35 sinistrómanos de ambos os sexos com idade entre os 15 e os 18 anos $(M=15.86 \pm .92)$. Empregou-se o Dutch H andedness Q uestionnaire (Van Strien, 1992) para avaliar a preferência manual e o Bassin A nticipation Timer para avaliar a antecipação-coincidência, a uma velocidade constante de $8 \mathrm{mph}$. Utilizaram-se três orientações do estímulo: da esquerda para a direita (ED), da direita para a esquerda (DE) e, no plano sagital. A ANOVA $2 \times 2 \times 3$ (preferência manual, sexo, direcção), com medidas repetidas no último factor, para um $\mathrm{p} \leq 0,05$, revelou que (i) todos os sujeitos obtiveram melhores resultados no plano sagital (ii) o factor preferência manual não apresentou efeitos significativos (iii) o sexo masculino foi mais preciso e menos variável do que o sexo feminino.

Palavras-chave: antecipação-coincidência, direcção do estímulo, preferência manual

\section{ABSTRACT \\ Manual preference in a coincidence-anticipation task: effects of varying the stimulus orientation}

The purpose of this study was to analyze the existing relation between performance in a coincidence-anticipation task, varying the orientation of the stimulus, manual preference and sex. Thirty one righthanders and 35 lefthanders of both sexes participated in this study $(M=15.86 \pm .92)$. The Dutch

Handedness Questionnaire was used (Van Strien, 1992) to evaluate manual preference and the Bassin Anticipation Timer to evaluate the coincidence-anticipation, at a constant speed of $8 \mathrm{mph}$. Subjects were positioned to view a stimulus runway approaching from their left (LR), right (RL), or directly from the front, in a sagittal plan (C). The ANOVA $2 \times 2 \times 3$ (manual preference, sex, direction of stimulus approach), with repeated measures in the last factor $(p \leq 0,05)$, indicated that (i) all subjects performed better in the sagittal plan (ii) handedness as factor didn't show statistical significance; (iii) males were more precise and less variable than females.

Key-words: coincidence-anticipation, stimulus approach, handedness 


\section{INTRODUÇÃO}

Em muitas actividades é crucial a coordenação temporal entre a condição de estímulo ambiental e a acção individual. Nestes casos, e independentemente da habilidade motora, o sucesso depende da antecipação temporal e da sincronização dos actos motores com o meio ambiente externo (35). As respostas que requerem precisão espacial e temporal são responsáveis pela performance nas tarefas de antecipaçãocoincidência (10), definida por Belisle em 1963 como a execução de uma resposta de movimento coincidente com a chegada de um estímulo a um determinado ponto de intersecção. A capacidade de antecipação-coincidência designa-se também por timing antecipatório $(16,31)$ ou timing de antecipação $(6,30)$. Poulton (26), num dos primeiros trabalhos sobre este assunto, apresentou uma estrutura teórica definindo três classes de antecipação distintas: a antecipação efectora, a antecipação receptora e a antecipação perceptiva. Na primeira, o sujeito deve prever o tempo de duração de execução do seu próprio movimento para que a sua resposta coincida com o acontecimento exterior. Na segunda, o indivíduo prevê a chegada do estímulo para o qual a resposta é dada (avalia a duração do evento externo). Na última, pretende-se que o executante deduza a natureza de futuros sinais através da sua experiência anterior. Neste caso, o executante deve fazer previsões espaciais e temporais através do padrão de regularidade dos estímulos. A integração da antecipação efectora com a receptora é denominada antecipação-coincidência.

Nas situações em que as respostas exigem antecipação, Schmidt (30) diferencia dois tipos. O primeiro refere-se à antecipação do tempo de chegada de um estímulo, permitindo ao sujeito realizar a sua resposta coincidentemente com o evento; o segundo envolve a coordenação do movimento de vários segmentos corporais na direcção e no tempo apropriados, para que o movimento resultante desta acção seja eficiente. Quando se começou a dar relevância ao estudo da antecipação-coincidência investigaram-se várias condições usando diferentes tarefas, mas foi a partir do momento em que o Bassin Anticipation Timer ficou disponível pela LaFayette Instrument Company, em 1976, que se uniformizaram procedimentos permitindo comparações de resultados. A pesquisa sobre a antecipação-coincidência tem desenvolvido esforços no sentido de descrever os aspectos inerentes à forma como se processa o desenvolvimento da resposta, bem como as variáveis que podem estar envolvidas e que afectam a performance e a aprendizagem da antecipação-coincidência. As variáveis independentes mais estudadas têm sido a idade (2, 9, $27,31)$, o sexo $(3,25,31,35,39)$, a velocidade do estímulo $(31,35)$, a complexidade da resposta motora $(9,39,40)$, os feedbacks $(4,27,39)$ e a prática $(11,12,13,31,34)$. No que diz respeito à primeira variável, tem sido demonstrado uma superioridade do sexo masculino em relação ao feminino $(3,15,31,39)$. A questão da preferência manual na execução de tarefas deste tipo tem sido muito pouco estudada. Normalmente são apenas consideradas amostras de indivíduos destrímanos (preferência manual direita).

Em muitas habilidades que requerem antecipaçãocoincidência, os estímulos podem aproximar-se do executante provindo de várias direcções. Payne (24) examinou a influência da direcção do estímulo na performance de uma tarefa de pressão sobre um botão, comparando a performance quando o estímulo se aproximava no plano sagital, pelo lado esquerdo e pelo lado direito. Os resultados demonstraram que quando o estímulo se aproximava no plano sagital, os erros eram significativamente menores do que quando se aproximava pelo lado direito ou pelo lado esquerdo. Coker (5), utilizando uma resposta motora mais complexa de batimento balístico (utilizando um taco de baseball), comparou a performance contrastando apenas a direcção do estímulo proveniente do lado direito e do lado esquerdo. Os resultados obtidos não evidenciaram diferenças estatisticamente significativas entre as duas direcções.

Um estímulo que se apresenta pelo lado esquerdo possui, segundo a literatura $(7,17,21,22,33)$ uma identificação perceptiva nas suas componentes bio-informacionais mais fácil do que o estímulo que se apresenta pelo lado direito.

De acordo com Nicholls e Roberts (23), esta polarização perceptiva para o lado esquerdo pode ser o resultado (a) de uma exploração polarizada da esquerda para a direita; (b) de uma activação prémotora do hemisfério direito, ou (c) de uma polarização atencional hemi-espacial esquerda. Os autores atrás citados investigaram a assimetria perceptiva de leitores ingleses e hebraicos numa tarefa de bissec- 
ção de uma linha. Foi-lhes pedido que seguissem um marcador enquanto este se movia da esquerda para a direita ou da direita para a esquerda, parando-o quando este alcançasse o ponto médio da linha. Ambos os grupos foram mais precisos a parar o marcador no ponto médio da linha na situação em que este se movia da esquerda para a direita. Os resultados deste estudo suportam a hipótese da polarização atencional no lado esquerdo.

Os efeitos dos hábitos de leitura na percepção de estímulos lateralizados têm sido investigados (7, 8, 22, 42), chegando-se à conclusão que existe uma tendência para orientar a atenção para o lado no qual, normalmente, a leitura se inicia. Esta observação decorre do facto que, na maioria das línguas europeias, a direcção da leitura e da escrita apresenta-se da esquerda para a direita. Assim, os leitores daquelas línguas farão a varredura em ambos os processos da esquerda para a direita.

Pode ser intuído que o sentido da leitura de uma pessoa terá um impacto quer em tarefas linguísticas quer óculo-motoras (42).

Nachshon (21) investigou os efeitos da lateralidade e dos hábitos de leitura na preferência direccional em sujeitos Israelitas destrímanos e sinistrómanos, cuja língua nativa era o hebraico. Os sujeitos identificaram e reproduziram disposições horizontais de estímulos visuais, que foram apresentadas ou simultaneamente (experiência I), ou sequencialmente (experiência II). Na experiência I, os sujeitos de ambos os grupos mostraram preferências direccionais similares, da esquerda para a direita. Na experiência II os destrímanos mostraram preferências da direita para a esquerda, enquanto que os sinistrómanos mostraram padrões inconsistentes de resposta. As diferenças entre os sexos revelaram-se somente entre os sinistrómanos. Os resultados foram interpretados como mostrando uma predominância de efeitos do hábito de leitura na experiência I, e de efeitos da lateralidade na experiência II. O autor concluiu que, dependendo das circunstâncias experimentais, as preferências direccionais podem ser uma função de hábitos de leitura, ou da lateralidade, ou de ambos.

Como a literatura, nos estudos que relacionam a capacidade de antecipação-coincidência com a preferência manual, é escassa, consideramos importante verificar até que ponto a direcção do estímulo numa tarefa de antecipação-coincidência está associada à preferência manual do indivíduo. Ao comparar destrímanos e sinistrómanos em tarefas de antecipaçãocoincidência, pretendemos também contribuir para a caracterização do comportamento motor de populações jovens. Neste estudo apontamos como objectivos a análise da relação existente entre a performance numa tarefa de antecipação-coincidência, variando a orientação do estímulo, a preferência manual e o sexo. Como no presente estudo ambos os grupos de sujeitos enquadram-se numa cultura ocidental, possuindo uma gramática da acção orientada da esquerda para a direita (e.g. nas acções da leitura e da escrita), pressupomos que a direcção ED proporcione os melhores resultados.

\section{MÉTODO}

\section{Sujeitos}

Participaram do estudo 66 estudantes de ambos os sexos (30 do sexo masculino e 36 do sexo feminino), com idade cronológica entre os 15 e os 18 anos $(M=15.86 \pm .926)$. Numa fase inicial foram seleccionados os alunos sinistrómanos, tendo como critério a mão preferida para escrever. De seguida, foram escolhidos aleatoriamente alunos destrímanos com características semelhantes aos sinistrómanos, nomeadamente no que respeita ao sexo e à idade. Numa fase posterior, foram avaliados os sujeitos relativamente à sua preferência manual, desta vez de uma forma mais pormenorizada através da aplicação de um questionário (Dutch $\mathrm{H}$ andedness Q uestionnaire, 36). Assim, foram seleccionados 31 destrímanos e 35 sinistrómanos.

\section{Instrumento e tarefa}

$O$ instrumento utilizado nesta pesquisa foi o Bassin Anticipation Timer da Lafayette Instruments, modelo n. ${ }^{\circ}$ 50 575. A tarefa consistiu em apertar o interruptor em coincidência com a incandescência do último díodo. O resultado (em cada tentativa) foi apresentado em milisegundos.

\section{Delineamento e Procedimentos}

Os sujeitos foram testados individualmente num banco de altura regulável. Para garantir o ângulo de apresentação do estímulo, a relação entre a altura do banco e a estatura do sujeito foi ajustada, utilizando- 
se para tal uma recta localizada numa parede paralela à calha do instrumento, demarcando $25^{\circ}$ em relação à incidência no último díodo. Nas duas primeiras condições, o banco foi colocado a $1,5 \mathrm{~m}$ da calha do Bassin, equidistante das suas extremidades. $\mathrm{Na}$ primeira condição, o estímulo propagava-se da esquerda para a direita (ED) e na segunda, da direita para a esquerda (DE). Na terceira condição, o banco estava posicionado na continuação da última calha $\mathrm{e}$ a 1,5m desta, de forma a que a propagação do estímulo se apresentasse no plano sagital. Os sujeitos foram informados sobre o objectivo da tarefa: fazer coincidir a resposta com o acendimento do último díodo da calha. Para cada uma das três condições referidas, foram realizados 20 ensaios com a mão preferida, precedidos de um ensaio de contacto, não avaliado, em cada condição. Cada sujeito efectuou um total de 60 tentativas a uma velocidade constante do estímulo de $357 \mathrm{~cm} / \mathrm{s}$ ( $8 \mathrm{mph}$ ), com um intervalo inter-tentativas de 10s. O sinal de aviso (díodo amarelo) mantinha-se aceso durante $500 \mathrm{~ms}$ antes do início da propagação do estímulo. Foi fornecido conhecimento dos resultados incluindo amplitude e a direcção de erro, após cada ensaio.

\section{RESULTADOS}

Foram calculados os erros absoluto (EA), constante (EC) e variável (EV) para cada participante. As variáveis dependentes foram analisadas através de

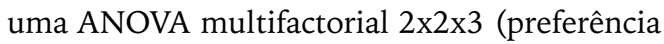
manual, sexo, direcção), com medidas repetidas no último factor. O teste post hoc utilizado foi o de Bonferroni. O nível de significância para a rejeição da hipótese nula foi fixado em $\mathrm{p} \leq 0,05$.

\section{Erro absoluto}

No Quadro 1 apresentam-se os valores da média e desvio-padrão do EA nas três direcções do estímulo, em função do sexo e da preferência manual. O EA informa-nos sobre a precisão do erro, em módulo.

Quadro 1. Valores das médias e dos desvios-padrão do EA (ms] nas três direcções do estímulo, em função do sexo e da preferência manual.

\begin{tabular}{lcccccc}
\hline & & Destrímanos & & \multicolumn{3}{c}{ Sinistrómanos } \\
& Masculino & $\begin{array}{c}\text { Feminino } \\
\text { Feminino }\end{array}$ & TOTAL & Masculino & \\
\hline ED & $38.32 \pm 11.6$ & $45.94 \pm 10.41$ & $42.01 \pm 11.5$ & $33.67 \pm 7.6$ & $49.89 \pm 13.9$ & $43.69 \pm 14.2$ \\
C & $29.10 \pm 6.0$ & $29.92 \pm 7.7$ & $29.50 \pm 6.7$ & $22.61 \pm 7.2$ & $32.15 \pm 9.2$ & $28.50 \pm 9.6$ \\
DE & $37.06 \pm 9.1$ & $40.32 \pm 12.1$ & $38.64 \pm 10.6$ & $29.26 \pm 8.6$ & $49.50 \pm 19.0$ & $41.76 \pm 18.6$ \\
\hline
\end{tabular}

O factor direcção teve um efeito significativo $[\mathrm{F}(2$, $60)=54.997, p=.000]$. A apresentação no plano sagital proporcionou o melhor desempenho, apresentando EA inferiores, tanto em destrímanos como em sinistrómanos. O teste post hoc revelou diferenças estatisticamente significativas apenas entre a direcção $\mathrm{C}$ e as restantes direcções ( $\mathrm{p}$.05). Foi verificado um efeito significativo da variável sexo $[\mathrm{F}(1$, $61)=18.490, \mathrm{p}=.000$ ], apontando para uma maior precisão no sexo masculino. A interacção sexo e direcção também evidenciou um efeito significativo $[\mathrm{F}(2,60)=4.202, \mathrm{p}=.020]$, obtendo os rapazes na direcção central o melhor desempenho e as raparigas na direcção ED o pior. O factor preferência manual não demonstrou ter um efeito significativo $[\mathrm{F}(9$, $53)=1.462, p=.187]$. Em nenhuma outra interacção se verificaram efeitos significativos.

\section{Erro constante}

No Quadro 2 apresentam-se os valores da média e desvio-padrão do EC nas três direcções do estímulo, em função do sexo e da preferência manual. O EC informa-nos sobre a direcção e a magnitude do erro, isto é, se resposta foi antecipada (valores negativos) ou se foi atrasada (valores positivos). 
Quadro 2. Valores das médias e dos desvios-padrão do EC nas três direcções do estímulo, em função do sexo e da preferência manual.

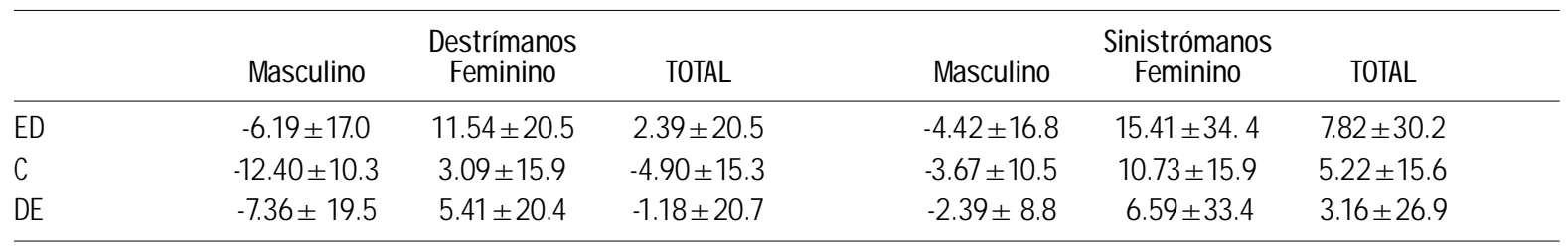

Verificou-se um efeito significativo em relação à variável sexo $[\mathrm{F}(1,61)=10.561, \mathrm{p}=.002]$. As raparigas atrasaram as suas respostas e os rapazes anteciparam. Nenhum outro factor ou interacção demonstrou efeitos significativos.

\section{Erro variável}

No Quadro 3 apresentam-se os valores da média e desvio-padrão do EC nas três direcções do estímulo, em função do sexo e da preferência manual. O EC fornece-nos informações sobre a variabilidade das respostas no tempo, ou seja, sobre a consistência do comportamento motor ao longo das tentativas.

Quadro 3. Valores das médias e dos desvios-padrão do EV nas três direcções do estímulo, em função do sexo e da preferência manual.

\begin{tabular}{lcccccc}
\hline & Masculino & $\begin{array}{c}\text { Destrímanos } \\
\text { Feminino }\end{array}$ & TOTAL & Masculino & $\begin{array}{c}\text { Sinistrómanos } \\
\text { Feminino }\end{array}$ & TOTAL \\
\hline ED & $46.23 \pm 13.9$ & $53.47 \pm 9.1$ & $49.73 \pm 12.2$ & $40.47 \pm 9.1$ & $49.18 \pm 14.0$ & $45.85 \pm 12.9$ \\
C & $34.00 \pm 4.9$ & $34.20 \pm 8.9$ & $34.10 \pm 7.0$ & $27.29 \pm 9.7$ & $34.92 \pm 8.0$ & $32.01 \pm 9.3$ \\
DE & $44.80 \pm 13.0$ & $48.51 \pm 13.9$ & $46.60 \pm 13.3$ & $35.90 \pm 9.8$ & $51.69 \pm 12.3$ & $45.65 \pm 13.7$ \\
\hline
\end{tabular}

O factor direcção teve um efeito significativo $[\mathrm{F}(2$, $60)=53.292, p=.000]$. A apresentação no plano sagital proporcionou desempenhos menos variáveis do que as restantes direcções. O teste post hoc revelou diferenças estatisticamente significativas apenas entre a direcção $\mathrm{C}$ e as restantes direcções ( $\mathrm{p} \leq .05)$. Foi verificado um efeito significativo da variável sexo $[\mathrm{F}(1,61)=14.713, \mathrm{p}=.000]$, apontando para menor variabilidade no sexo masculino. O factor preferência manual não demonstrou ter um efeito significativo $[F(1,61)=3.717, p=.059]$. Nenhum outro factor ou interacção demonstrou efeitos significativos.

De uma forma geral, os resultados deste estudo demonstraram que (i) tanto destrímanos como sinistrómanos obtiveram melhores resultados quando o estímulo se deslocava no plano sagital e apresentaram os piores resultados na direcção ED; (ii) o factor preferência manual não apresentou efeitos significativos (iii) os indivíduos do sexo masculino apresentaram desempenhos superiores aos do sexo feminino, tanto na precisão como na variabilidade; (iv) os rapazes anteciparam as suas respostas e as raparigas atrasaram.

\section{DISCUSSÃO}

Comparando a performance dos sujeitos nas três direcções do estímulo poderemos concluir que independentemente da preferência manual e do sexo, os sujeitos têm melhor desempenho quando o estímulo se desloca no plano sagital, confirmando os resultados obtidos por Payne (24). A hipótese colocada inicialmente não foi comprovada, uma vez que tanto em destrímanos como em sinistrómanos não se observaram diferenças estatisticamente significativas entre as direcções ED e DE, corroborando os resultados de Coker (5). É de salientar que estes autores utilizaram tarefas de diferente complexidade, sendo a de Payne (24) semelhante à utilizada neste estudo e a de Coker (5) mais complexa.

De forma a optimizarem os desempenhos dos sujeitos das suas amostras, talvez estes resultados possam contribuir para que, futuramente, os investigadores que utilizarem o Bassin A nticipation Timer empreguem o aparelho no plano sagital. Esta nossa sugestão baseia-se na constatação de que muitos estudos $(14,18$, $19,20,28,29,391,40)$ que utilizam este instrumento o colocam de forma a que o estímulo se desloque na 
direcção ED. Compreende-se que, em alguns dos trabalhos referidos anteriormente, esta direcção seja utilizada com o intuito de recriar situações similares às modalidades praticadas pelos sujeitos em estudo (19, 20, 29). Por outro lado, é natural o recurso às três direcções quando se pretende estudar o comportamento motor dos indivíduos de diferentes sexos, idades, experiências ou preferência manual. Nos estudos em que se pretende apenas avaliar o desempenho numa direcção do estímulo, os nossos resultados sugerem a direcção C, como já referimos.

No que respeita ao sexo, verificou-se uma superioridade dos indivíduos do sexo masculino em relação aos do sexo feminino, estando os nossos resultados em conformidade com os observados em outros estudos $(3,14,16,25,31)$. Tal como referem Wrisberg e colaboradores (34), até à adolescência pouca literatura revela uma superioridade de um sexo em relação ao outro; no entanto, entre sujeitos pós-púberes a diferença mais marcante entre os sexos tem demonstrado uma superioridade na performance do sexo masculino em tarefas que fazem uso da velocidade e da amplitude da resposta. Alguns autores $(26,30)$ observaram tempos de reacção mais rápidos no sexo masculino em tarefas de velocidade de reacção simples e complexa, tornando plausível esta sugestão. Tanto a velocidade de reacção como a antecipação-coincidência são capacidades do processamento da informação que exigem uma precisão espaço-temporal. Estas capacidades são reguladas pelo hemisfério direito (1) e onde se tem observado uma maior especialização hemisférica em rapazes do que em raparigas (37). Como, por sua vez, este hemisfério controla com maior habilidade a execução dos movimentos com a mão esquerda, poder-se-à supor que os melhores resultados obtidos pelos rapazes sinistrómanos sejam fruto da natureza na tarefa.

Seria interessante, em estudos posteriores, investigar, para além da mão preferida, o comportamento da mão não preferida nestes dois grupos de preferência manual, de forma a clarificar o papel da natureza da tarefa em situações semelhantes às descritas anteriormente.

\section{CORRESPONDÊNCIA \\ Paula Cristina Rodrigues}

Rua Dr. José Marinho, n 267, 4460-752 Custóias, Matosinhos

Portugal

Telefone: +35122 9545300; +351918808119

e-mail: packn@ clix.pt 


\section{REFERÊNCIAS}

1. Barthelemy S, Boulinguez P (2001). Manual reaction time asymmetries in human subjects: The role of movement planning and attention. N euroscience Letters 315(1-2): 41-44.

2. Borod JC, Caron HS, Koff E (1984). Left-handers and right-handers compared on performance and preference measures of lateral dominance. British Journal of Psychology 75 ( Pt 2): 177-186.

3. Brady F. (1996). Anticipation of coincidence, gender, and sports classification. Perceptual and M otor Skills 86: 227-239.

4. Brison T, Alain C (1996). Should common optimal movement patterns be identified as the criterion to be achieved? Journal of M otor Behavior 28: 211-223.

5. Coker C (2003). Influence of the direction of an approaching stimulus on coincident timing. Research Q uarterly for Exercise and Sport 74 (suppl. 1): A-29.

6. Dorfman P (1977). Timing and anticipation: a developmental perspective. Journal of $M$ otor Behavior 9: 67-79.

7. Eviatar Z (1995). Reading direction and attention: Effects on lateralized ignoring. Brain Cogn 29(2): 137-150.

8. Fagard J, Dahmen R (2003). The effects of reading-writing direction on the asymmetry of space perception and directional tendencies: A comparison between french and tunisian children. Laterality 8(1): 39-52.

9. Ferraz O (1993). Desenvolvimento de "timing" antecipatório em crianças. Revista Paulista de Educação Física 7(1): 13-44.

10. Fleury M, Bard C (1985). Age, stimulus velocity and task complexity as determiners of coincident timing behaviour. Journal of Human M ovement Studies 11: 305-317.

11. Freudenheim A, Tani G (1995). Efeitos da estrutura de prática variada na aprendizagem de uma tarefa de "timing" coincidente em crianças. Revista Paulista de Educação Física 9(2): 30-44.

12. Freudenheim AM, Tani G (1993). Formação de esquema motor em crianças numa tarefa que envolve timing coincidente. R evista Paulista de Educação Física 7(1): 30-44.

13. Freudenheim AM (1994). Um teste à formação de esquema: efeito da variabilidade e da quantidade da prática na produção de movimentos novos em adultos. R evista Paulista de Educação Física 8(1): 3-16.

14. Hart MA, Reeve TG (1997). A preliminary comparison of stimulus presentation methods with the bassin anticipation timing task. Perceptual and M otor Skills 85(1): 344-346.

15. Kuhlman J, Beitel P (1992). Coincidence anticipation: possible critical variables. Journal of Sport Behavior 15: 91-105.

16. Magill RA (1989). M otor learning: concepts and applications. $3^{a}$ ed.. Dubuque, Iowa, W. C. Brown.

17. Mattingley JB, Berberovic N, Corben L, Slavin MJ, Nicholls ME, Bradshaw JL (2004). The greyscales task: A perceptual measure of attentional bias following unilateral hemispheric damage. N europsychologia 42(3): 387-394.

18. McNevin N, Magill RA, Buekers MJ (1994). The effects of erroneous knowledge of results on transfer of anticipation timing. Research Quarterly for Exercise and Sport 65(4): 324-329.

19. Millslagle DG (2000). Dynamic visual acuity and coincidence-anticipation timing by experienced and inexperienced women players of fast pitch softball. Perceptual and M otor Skills 90(2): 498-504.

20. Molstad SM, Kluka DA, Love PA, Baylor KA, Covington NK, Cook TL (1994). Timing of coincidence anticipation by ncaa division i softball athletes. Perceptual and M otor Skills 79(3 Pt 2): 1491-1497.

21. Nachshon I (1983). Asymmetry in lateral directionality. Int J Neurosci 19(1-4): 191-203.

22. Nachson I, Hatta T (2001). Directional tendencies of hebrew, japanese, and english readers. Percept M ot Skills 93(1): 178-180.

23. Nicholls ME, Roberts GR (2002). Can free-viewing perceptual asymmetries be explained by scanning, pre-motor or attentional biases? Cortex 38(2): 113-136.

24. Payne VG (1988). Effects of direction of stimulus approach, eye dominance, and gender on coincidence anticipation timing performance. Journal of Human M ovement Studies 15: 17-25.

25. Petrakis E (1985). Sex differences and specificity of anticipation of coincidence. Perceptual and M otor Skills 61: 1135-1138.

26. Poulton E (1952). Perceptual anticipation in tracking with two-pointer and one-pointer displays. British Journal of Psychology 43(Part 3): 222-229.

27. Ramella R (1984). Effect of knowledge of results on anticipation timing by young children. Perceptual and M otor Skills 59: 519-525.

28. Ridenour MV (1981). Influence of background patterns of coincidence-anticipation performance. Perceptual and $M$ otor Skills 52(1): 47-50.

29. Ripoll H, Latiri I (1997). Effect of expertise on coincidenttiming accuracy in a fast ball game. Journal of Sports Sciences 15(6): 573-580.

30. Schmidt R (1968). Anticipation and timing in human motor performance. Psychological Bulletin 70: 631-646.

31. Stadulis R (1985). Coincidence-anticipation behavior of children. In J. Clark \& J. Humphrey (Eds.) M otor development: current selected Research Vol.1(pp.1-17). Princeton, N.J.: Princeton Book Co.

32. Tavares F, Vasconcelos O (1995). Tiempo de reacción simple e doble. Elección según el sexo y el predominio lateral en um grupo de escolares. In A ctas del Congreso Científico Olímpico 1992: A ctividade Física A daptada, Psicología y Sociologia. D eporte y D ocumentación, 24(1): (pp.299-303). Instituto Andaluz del Deporte, Málaga.

33. Taylor KI, Zach P, Brugger P (2002). Why is magical ideation related to leftward deviation on an implicit line bisection task? Cortex 38(2): 247-252.

34. Teixeira L, Paroli R (2000): Assimetrias laterais em acções motoras: preferências versus desempenho. M otriz 6(1): 1-8.

35. Teixeira L, Santos V, Andreysuk R (1992). Tarefas que envolvem timing antecipatório: seriam as velocidades mais baixas as mais fáceis para sincronizar?. R evista Paulista de Educação Física 6(2): 21-28.

36. Van Strien JW (1992). Classificatie van links - en rechtshangige proefperrsonen. Nederlands Tijdschrift voor de Psychologie 47: 88-92.

37. Van Vugt P, Fransen I, Creten W, Paquier P (2000). Line bisection performances of 650 normal children. N europsychologia 38(6): 886-895.

38. Welford AT (1980). Choice reaction time: basic concepts. In A.T. Welford (Ed.) Reaction times. (pp.73-128) London: Academic Press.

39. Williams L, Jasiewicz J (2001). Knowledge of results, movement type, and sex in coincidence timing. Perceptual and M otor Skills 92: 1057-1068.

40. Williams LR, Jasiewicz JM, Simmons RW (2001). Coincidence timing of finger, arm, and whole body movements. Perceptual and M otor Skills 92(2): 535-547.

41. Wrisberg C, Paul J, Ragsdale M (1979). Subject gender, Knowledge of results, and receptor anticipation. Research Quarterly 50: 699-708.

42. Zivotofsky AZ (2004). Choosing sides: Lateralization in line trisection and quadrisection as a function of reading direction and handedness. Brain Res Cogn Brain Res 20(2): 206-211. 